\title{
A NUMERICAL STUDY ON THE SCALE OF LAMINATED MICROSTRUCTURE WITH SURFACE ENERGY
}

\author{
ZHIPING LI \\ SCHOOL OF MATHEMATICAL SCIENCES, PEKING UNIVERSITY, \\ BEIJING 100871, P.R.CHINA
}

\begin{abstract}
A mathematical model is given to study how the twin width of the needle-like laminate is related to the twin length and the surface energy density in austenite-martensite phase transition. A numerical method based on the mesh transformation method is given, and numerical experiments are made to establish such a relationship for an elastic crystal model.
\end{abstract}

\section{INTRODUCTION}

Many elastic crystals are known to undergo austenitic-martensitic phase transitions across the transformation temperature, when the higher symmetric phase (austenite) above the transformation temperature transforms to the lower symmetric phase (martensite), which often consists of fine mixtures of variants and exhibits microstructure, below the transformation temperature, and vice versa. Mathematical models and analysis based on the energy minimization have been developed to study the equilibrium state of phase transformations and microstructures [1]-[4]. The length scale of the microstructure, that is the width of the twinning laminates, is found to depend on the length of the laminates and the surface energy density on the interfaces between the twins (see $[1,5,6]$ among many others).

In the present paper, a mathematical model for studying the scale of twinned laminated needle-like microstructures is established. The model is based upon minimizing the total potential energy which is considered to be the sum of the

1991 Mathematics Subject Classification. 73C50,65K10,65N30.

Key words and phrases. non-quasiconvex energy, laminated microstructure, needle-like microstructure, surface energy, scale, mesh transformation method.

The research was supported in part by the Special Funds for Major State Basic Research Projects (G1999032802), NSFC and RFDP of China. 
elastic energy of needles near the austenite-finely twinned martensite interface and the surface energy. In our model, the average elastic energy density, i.e. the average elastic energy per volume, of the needles near the austenite-finely twinned martensite interface is considered to be a function of the needle length and the length scale of the twin width of the laminated needle-like microstructure, which is established numerically by applying the mesh transformation method (see [7, $8,9,10,11])$ to a specially designed boundary value problem to modelling the needle-like microstructures near the austenite-finely twinned martensite interface.

The choice of the mesh transformation method comes naturally from the observation that the numerical solutions of finite element approximations to the martensitic microstructures depend heavily on the mesh and a fixed mesh usually produces poor numerical results and can even lead to pseudo-microstructures $[7,12,13,14,15]$. The idea of the mesh transformation method is to involve the mesh distribution into the minimization procedure so that the mesh can align with the twin boundaries and thus essentially avoid the mesh dependence of the finite element approximation. Successful applications of the mesh transformation method can be found in numerical computations on simple laminated microstructures and needle-like microstructures $[8,9,10,11]$. Furthermore, the application of the mesh transformation method makes it possible for us to get numerical results with reasonable precision on a coarse mesh.

The rest of the paper is arranged as follows. In section 2, a 2-dimensional mathematical model for elastic crystals, which we use in our numerical experiments in the present paper, is presented. In section 3, a mathematical model for laminated needle-like microstructures with surface energy is given. In section 4, the mesh transformation method is described. In section 5, numerical examples are presented and the numerical results are given and discussed.

\section{A MATHEMATICAL MODEL FOR ELASTIC CRYSTALS}

In the well known geometrically nonlinear theory of martensitic microstructures given by Ball and James [1, 2], microstructures are characterized by energy minimizing sequences which consist of increasingly fine oscillations and lead to Young measures of gradients [16]. For the static problem of austenitic-martensitic phase transitions, the theory leads to the consideration of minimizing an integral functional

$$
F(u ; \Omega)=\int_{\Omega} f(\nabla u(x), \theta(x)) d x
$$


in a set of admissible functions

$$
\mathbb{U}\left(u_{0} ; \Omega\right)=\left\{u \in W^{1, p}\left(\Omega ; R^{m}\right): u=u_{0}, \text { on } \partial \Omega_{0}\right\},
$$

where $\Omega \subset R^{n}$ is a bounded open set with a Lipschitz continuous boundary $\partial \Omega, \partial \Omega_{0}$ is a subset of $\partial \Omega$ with a positive $(n-1)$-dimensional measure, $\theta$ is a given temperature field and $1<p<\infty$, and where the Ericksen-James elastic energy density $f(\cdot, \theta)$ is such that $f(\cdot, \theta)$ has a unique potential well above the transformation temperature $\left(\theta>\theta_{T}\right)$ and has several symmetry related potential wells below the transformation temperature $\left(\theta<\theta_{T}\right)[1,2,19,18]$. Since the Ericksen-James elastic energy density $f(\cdot, \theta)$ is required to be frame indifferent, it must be of the form $[17,18]$

$$
f(\nabla u, \theta)=\Phi(C, \theta),
$$

where $C=(\nabla u)^{T} \nabla u \in \mathbb{S}^{n}=\left\{A \in R^{n \times n}: A^{T}=A\right\}$, which is the set of symmetric matrices in $R^{n}$, is the right Cauchy-Green strain tensor.

In the present paper, we consider a two dimensional model $(n=m=2)$ with

$$
\begin{aligned}
\Phi(C, \theta)=\frac{b(\theta)}{4}\left(C_{11}-C_{22}\right)^{2}- & \frac{c(\theta)}{8}\left(C_{11}-C_{22}\right)^{2}\left|C_{11}-C_{22}\right| \\
& +\frac{d(\theta)}{16}\left(C_{11}-C_{22}\right)^{4}+e C_{12}^{2}+g(\operatorname{tr} C-2)^{2},
\end{aligned}
$$

where

$$
\begin{aligned}
& b(\theta)=\left(1+\alpha \arctan \mu\left(\theta-\theta_{T}\right)\right) d_{0} \hat{\varepsilon}^{2}, \\
& c(\theta)=2\left(1+\frac{1+2 \gamma}{3} \alpha \arctan \mu\left(\theta-\theta_{T}\right)\right) d_{0} \hat{\varepsilon}, \\
& d(\theta)=\left(1+\gamma \alpha \arctan \mu\left(\theta-\theta_{T}\right)\right) d_{0},
\end{aligned}
$$

and where $d_{0}>0, e>0$ and $g>0$ are the elastic moduli, $\hat{\varepsilon}$ is the transformation strain, $\theta_{T}$ is the transformation temperature,

$$
\alpha \approx \frac{2}{\pi}, \mu>0, \text { and } \gamma<1
$$

are the material constants used to reflect the change of elastic moduli and the energy barriers as the temperature varies.

Lemma 2.1. Let $\Phi(C, \theta)$ be defined by (2.4), let

$$
C_{\varepsilon, \delta, \gamma}=\left(\begin{array}{cc}
1+2 \varepsilon+\delta & \gamma \\
\gamma & 1+\delta
\end{array}\right)
$$


and let $C_{\varepsilon}=C_{\varepsilon,-\varepsilon, 0}$. Then, we have

$$
\Phi\left(C_{\varepsilon}, \theta\right) \leq \Phi\left(C_{\varepsilon, \delta, \gamma}, \theta\right), \quad \forall \varepsilon, \delta, \gamma \in R^{1}
$$

and the equality holds if and only if $\delta=-\varepsilon$ and $\gamma=0$.

Proof. A direct calculation by the definition of $\Phi$ gives

$$
\Phi\left(C_{\varepsilon, \delta, \gamma}, \theta\right)=\Phi\left(C_{\varepsilon}, \theta\right)+e \gamma^{2}+2 g(\delta+\varepsilon)^{2} .
$$

Since $e>0$ and $g>0$, the lemma follows.

Lemma 2.2. Let $\Phi(C, \theta)$ be defined by (2.4), then

$$
\Phi\left(C_{0}, \theta\right) \leq \Phi(C, \theta), \quad \forall C \in \mathbb{S}^{2}, \quad \text { if } \theta>\theta_{T},
$$

where the equality holds if and only if $C=C_{0}$ which is the identity matrix $I$, and

$$
\Phi\left(C_{ \pm \hat{\varepsilon}}, \theta\right) \leq \Phi(C, \theta), \quad \forall C \in \mathbb{S}^{2}, \quad \text { if } \theta<\theta_{T},
$$

where the equality holds if and only if $C=C_{ \pm \hat{\varepsilon}}$.

Proof. Since all $C \in \mathbb{S}^{2}$ can be written in the form of $(2.9)$ and since $\Phi\left(C_{-\varepsilon}, \theta\right)=$ $\Phi\left(C_{\varepsilon}, \theta\right)$, by lemma 2.1, it only needs to show that (2.12) and (2.13) are satisfied for all $C=C_{\varepsilon}$ with $\varepsilon \geq 0$ for which we have

$$
\Phi\left(C_{\varepsilon}, \theta\right)=b(\theta) \varepsilon^{2}-c(\theta) \varepsilon^{3}+d(\theta) \varepsilon^{4} .
$$

Thus, by direct calculations on (2.14), it is easily verified that the conclusions of the theorem is true.

Figure 1 shows $\Phi\left(C_{\varepsilon}, \theta\right)$ as a function of $\varepsilon$ for $\alpha=2.02 / \pi, \mu=0.25, \gamma=0$, $\hat{\varepsilon}=0.05, d_{0}=500$ and various $\theta$.

Theorem 2.1. Let $\Phi(C, \theta)$ be given by (2.4). Then the energy density $f(\nabla u, \theta)$ defined by (2.3) has

(i): a unique potential well $S O(2)$ for $\theta>\theta_{T}$;

(ii): two symmetry related rotationally invariant potential wells $S O(2) U_{0}$ and $S O(2) U_{1}$ for $\theta<\theta_{T}$,

where $S O(2)$ is the set of all $2 \times 2$ rotational matrices, and

$$
U_{0}=\left(\begin{array}{cc}
\sqrt{1-\hat{\varepsilon}} & 0 \\
0 & \sqrt{1+\hat{\varepsilon}}
\end{array}\right), \quad U_{1}=\left(\begin{array}{cc}
\sqrt{1+\hat{\varepsilon}} & 0 \\
0 & \sqrt{1-\hat{\varepsilon}}
\end{array}\right) .
$$




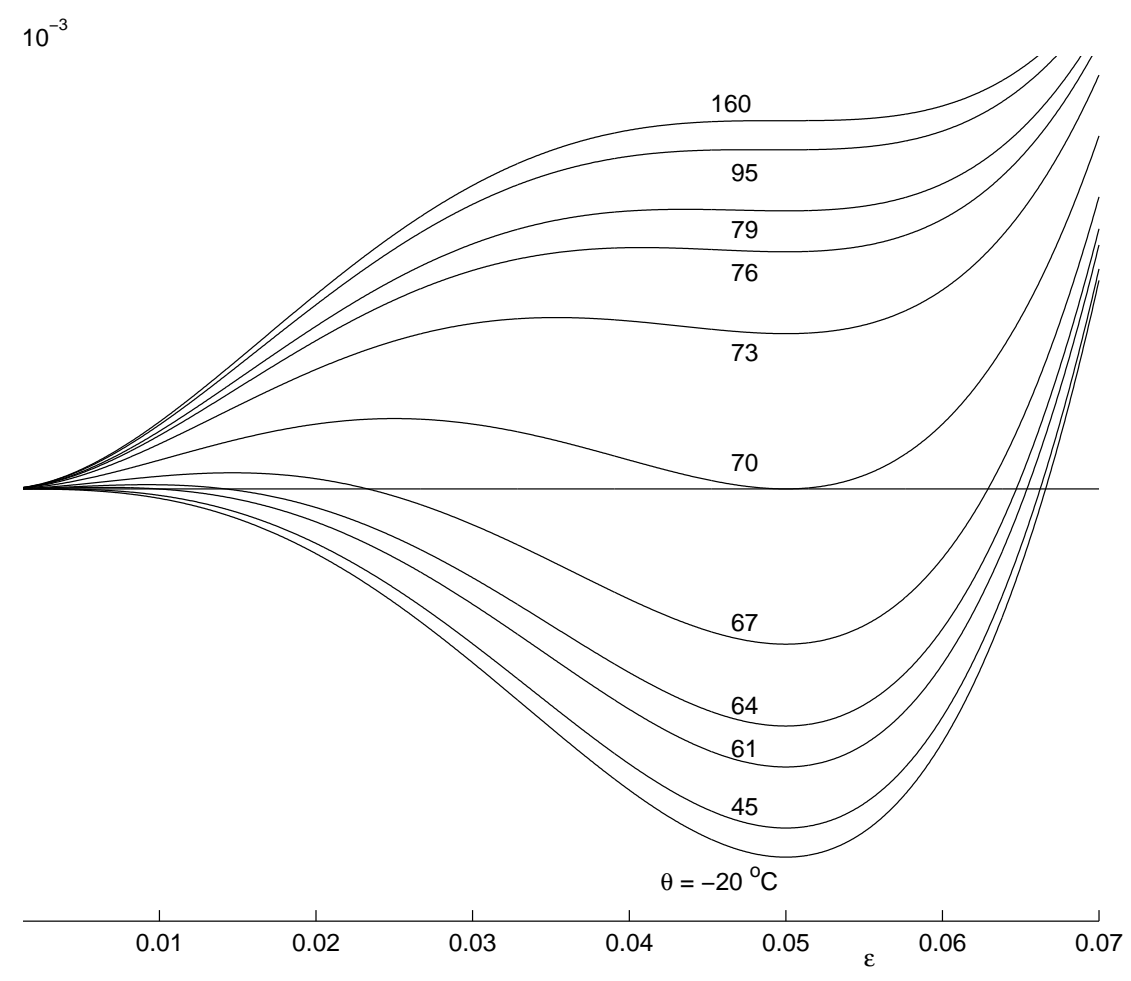

Figure 1. The energy density $\Phi\left(C_{\varepsilon}, \theta\right)$.

Furthermore, $U_{0}$ and $R^{ \pm} U_{1}$ are in rank-one connection. More precisely, let $\eta_{1}=$ $\sqrt{1-\hat{\varepsilon}}$ and $\eta_{2}=\sqrt{1+\hat{\varepsilon}}$ and let

$$
R^{ \pm}=\left(\begin{array}{cc}
\eta_{1} \eta_{2} & \pm \hat{\varepsilon} \\
\mp \hat{\varepsilon} & \eta_{1} \eta_{2}
\end{array}\right)
$$

then, we have

$$
R^{ \pm} U_{1}=U_{0}+\mathbf{a}^{ \pm} \otimes \mathbf{n}^{ \pm},
$$

where $\mathbf{a}^{ \pm}=\sqrt{2} \hat{\varepsilon}\left(\eta_{1}, \mp \eta_{2}\right)^{T}$ and $\mathbf{n}^{ \pm}=\frac{1}{\sqrt{2}}(1, \pm 1)^{T}$.

Proof. The conclusions (i) and (ii) follow directly from Lemma 2.2. The rest of the theorem can be verified by direct calculations (c.f. [1, 14]).

The elastic energy density $\Phi(C, \theta)$ given above, of which the elastic moduli do not linearly depend on the temperature $\theta$ (compare [18]), has the advantages that (i) the temperature range during which the the transformation takes place can be adjusted by the parameter $\mu$, the greater the $\mu$ the smaller the temperature 
range, (ii) the magnitude of the energy barriers for the phase transitions can be adjusted by the parameters $\alpha$ and $\gamma$, and (iii) the locations of the energy wells remain unchanged as the temperature varies.

While the energy minimizing sequences take a crucial role in the computation of Young measures which give the probability distribution of the microstructure, the local minimizers of the the energy $F(u ; \Omega)$ in $\mathbf{U}\left(u_{0} ; \Omega\right)$ can also be of great importance, especially in the case, as is in this paper, when the profile of the needles near the austenite-finely twinned martensite interface is under consideration. In fact, it is such local minimizers, which contain the detailed information of the needles near the austenite-finely twinned martensite interface, that we are trying to model and compute in this paper.

\section{A mathematical MOdel FOR NEEDLES With SURFACE ENERGy}

At the transformation temperature $\left(\theta=\theta_{T}\right)$, the martensitic and austenitic phases may coexist, and in such a case the martensitic twins are observed to bend into needles as they approach the austenite-twinned-martensite interface. It is believed in some theories that the length scale of the width of the twinned laminates is determined by the balance of the elastic energy introduced by the bending of the twins near the austenite-twinned-martensite interface and the surface energy which is defined on the interfaces of the twins $[1,2,5,6]$. In the following, we are going to derive a computational model for this approach.

Without loss of generality, we may assume that the gradients of the martensitic twins are $U_{0}$ and $R^{-} U_{1}$, the volume fractions are $(1-\lambda)$ and $\lambda$ respectively, and thus the average of deformation gradients is $A_{\lambda}^{-}=(1-\lambda) U_{0}+\lambda R^{-} U_{1}$. Let

$$
\begin{aligned}
\xi^{ \pm} & =\arctan \frac{\lambda \hat{\varepsilon}\left(\eta_{1}+\eta_{2}\right)}{(1+\lambda \hat{\varepsilon}) \eta_{1}+(1-\lambda \hat{\varepsilon}) \eta_{2}} \pm \arctan \frac{\hat{\varepsilon} \sqrt{1+4 \lambda(\lambda-1)}}{1+\eta_{1} \eta_{2}}, \\
\varphi^{ \pm} & =\arctan \frac{(1-\lambda \hat{\varepsilon}) \eta_{2}-\cos \xi^{ \pm}}{\lambda \hat{\varepsilon} \eta_{2}-\sin \xi^{ \pm}},
\end{aligned}
$$

where $\hat{\varepsilon}$ is the transformation strain and $\eta_{1}=\sqrt{1-\hat{\varepsilon}}, \eta_{2}=\sqrt{1+\hat{\varepsilon}}$ (see section 2). Then, it is easily verified that $A_{\lambda}^{-}$is rank-one connected to the austenitic potential well $S O(2)$ at exactly two rotational matrices

$$
R\left(\xi^{ \pm}\right)=\left(\begin{array}{cc}
\cos \xi^{ \pm} & -\sin \xi^{ \pm} \\
\sin \xi^{ \pm} & \cos \xi^{ \pm}
\end{array}\right)
$$


and for some vectors $\mathbf{b}^{ \pm} \in R^{2}$,

$$
A_{\lambda}^{-}-R\left(\xi^{ \pm}\right)=\mathbf{b}^{ \pm} \otimes \mathbf{n}\left(\varphi^{ \pm}\right)
$$

where $\mathbf{n}(\varphi)=(\cos \varphi, \sin \varphi)^{T}$. Therefore, the austenite-twinned-martensite interface has to be normal to either $\mathbf{n}\left(\varphi^{+}\right)$or $\mathbf{n}\left(\varphi^{-}\right)$. Noticing that $\mathbf{n}^{-}=\mathbf{n}(-\pi / 4)$, we see that the periodic relationship

$$
u\left(x+\frac{w \mathbf{n}\left(\varphi^{ \pm}+\pi / 2\right)}{\cos \left(\pi / 4-\varphi^{ \pm}\right)}\right)-A_{\lambda}^{-}\left(x+\frac{w \mathbf{n}\left(\varphi^{ \pm}+\pi / 2\right)}{\cos \left(\pi / 4-\varphi^{ \pm}\right)}\right)=u(x)-A_{\lambda}^{-}(x), \quad \forall x \in R^{2}
$$

holds for both the deformation of the twinned martensite with the twin width $w$ and the deformation of the austenite $R\left(\xi^{ \pm}\right) x$. This periodicity allows us to consider a periodic problem on a parallelogram with one pair of parallel sides, of which the length is the multiple of $\frac{w \mathbf{n}\left(\varphi^{ \pm}+\pi / 2\right)}{\cos \left(\pi / 4-\varphi^{ \pm}\right)}$, perpendicular to $\mathbf{n}\left(\varphi^{ \pm}\right)$and the other pair of parallel sides perpendicular to $\mathbf{n}^{-}$.

Let $\Omega$ be such a parallelogram centered at 0 with the length of the sides perpendicular to $\mathbf{n}^{-}$being $l_{0}$. Let the boundary conditions $u_{n}$ be given by

$$
u_{n}(x)= \begin{cases}u_{n}\left(x \mp w \mathbf{n}^{-}\left(\varphi^{-}\right)\right), & \text {on } \partial \Omega^{ \pm}, \\ U_{0}\left(x-w\left(\frac{1}{2}-\lambda\right) \mathbf{n}^{-}\right)+\left(\int_{0}^{w^{-1} x \cdot \mathbf{n}^{-}} \sigma_{\lambda}(s) d s\right) \mathbf{a}^{-}, & \text {on } \partial \Omega_{-}, \\ R\left(\xi^{-}\right)\left(x-w\left(\frac{1}{2}-\lambda\right) \mathbf{n}^{-}\right), & \text {on } \partial \Omega_{+}\end{cases}
$$

where $\partial \Omega^{ \pm}$are the pair of sides perpendicular to $\mathbf{n}^{-}, \partial \Omega_{ \pm}$are the pair of sides perpendicular to $\mathbf{n}\left(\varphi^{-}\right)$, and

$$
\sigma_{\lambda}(s)= \begin{cases}0, & k-\lambda \leq s-\frac{1}{2}<k, \quad \forall k \in \mathfrak{I} \\ 1, & k-1 \leq s-\frac{1}{2}<k-\lambda, \quad \forall k \in \mathfrak{I}\end{cases}
$$

where $\mathfrak{I}$ is the set of all integers. It is easily seen $[1,14]$ that $u_{n}$ defines a pair of twin laminate on $\partial \Omega_{-}$and an austenite on $\partial \Omega_{+}$(see figure 2 ).

The domain $\Omega$ is said to have an admissible decomposition for needles, if $\Omega$ can be divided into three disjoint connected subdomains $\Omega_{i}, i=0,1,2$ with Lipschitz continuous boundaries $\partial \Omega^{i}=\partial \Omega_{i} \backslash \partial \Omega_{-}, i=1,2$, each of which consists of two single valued monotone curves as shown in figure 2 . Let the set of admissible functions $U_{n}\left(u_{n} ; \Omega\right) \subset U\left(u_{n} ; \Omega\right)$ be the set of all functions $u \in U\left(u_{n} ; \Omega\right)$ such that the sets

$$
\begin{aligned}
& \Omega^{0}=\left\{x \in \Omega:\left|\nabla u(x)-R\left(\xi^{-}\right)\right|<\left|\nabla u(x)-U_{0}\right| \wedge\left|\nabla u(x)-R^{-} U_{1}\right|\right\}, \\
& \Omega^{1}=\left\{x \in \Omega:\left|\nabla u(x)-U_{0}\right|<\left|\nabla u(x)-R^{-} U_{1}\right| \wedge\left|\nabla u(x)-R\left(\xi^{-}\right)\right|\right\},
\end{aligned}
$$




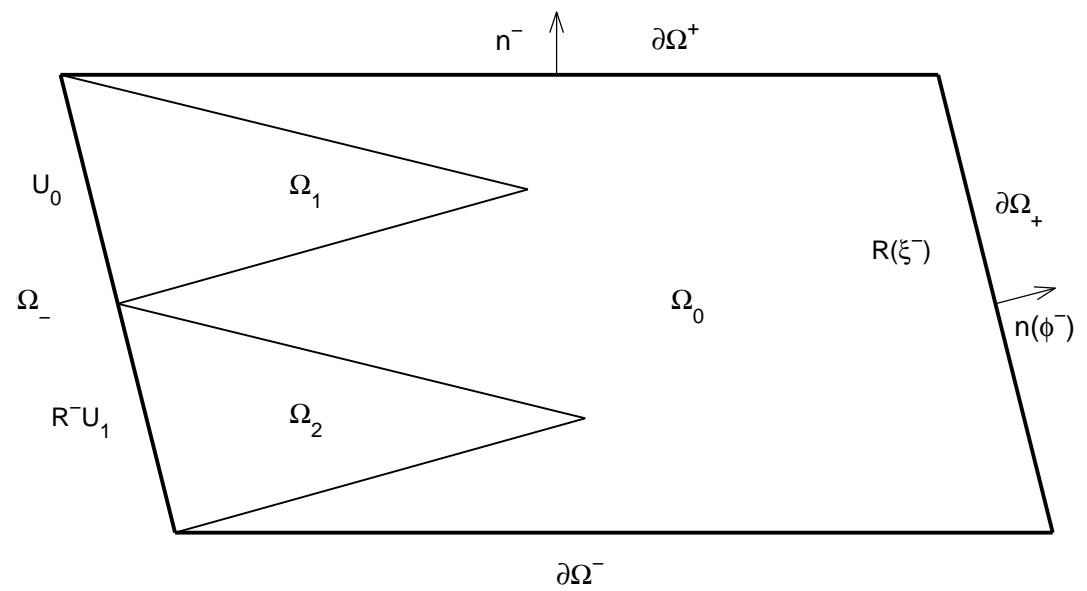

Figure 2. Admissible decomposition of $\Omega$ for needles and $\nabla u_{n}$ on $\partial \Omega_{ \pm}$.

$$
\Omega^{2}=\left\{x \in \Omega:\left|\nabla u(x)-R^{-} U_{1}\right|<\left|\nabla u(x)-U_{0}\right| \wedge\left|\nabla u(x)-R\left(\xi^{-}\right)\right|\right\},
$$

where $\alpha \wedge \beta=\min \{\alpha, \beta\}$, give an admissible decomposition of $\Omega$ for needles.

For the elastic energy of needles, we consider the problem

$$
\left\{\begin{array}{l}
\text { find } u \in U_{n}\left(u_{n} ; \Omega\right) \text { such that } \\
F(u ; \Omega)=\inf _{v \in U_{n}\left(u_{n} ; \Omega\right)} F(v ; \Omega) .
\end{array}\right.
$$

Basically, the problem (3.6) is to find the energy minimizer among all possible needles. Denote the elastic energy of a twin needles $u \in U_{n}\left(u_{n} ; \Omega\right)$ by $E_{n}\left(l_{0}, w, u\right)$, and define the elastic energy of the minimizing twin needles by

$$
E_{n}\left(l_{0}, w\right)=\inf _{u \in U_{n}\left(u_{n} ; \Omega\right)} E_{n}\left(l_{0}, w, u\right)
$$

We have, by scaling $u_{\delta}(x)=\delta u\left(\delta^{-1} x\right)$, that

$$
E_{n}\left(\delta l_{0}, \delta w\right)=\delta^{2} E_{n}\left(l_{0}, w\right) .
$$

Denote $l_{n}\left(l_{0}, w, u\right)$ the total interface length of the two needles $\left(\cup_{i=1}^{2} \partial \Omega^{i}\right) \backslash$ $\partial \Omega_{-}$. Let $\left\{u^{(\alpha)}\right\} \subset U_{n}\left(u_{n} ; \Omega\right)$ be minimizing sequences of problem (3.6), define

$$
l_{n}\left(l_{0}, w\right)=\inf _{\left\{u^{(\alpha)}\right\}} \liminf _{\alpha \rightarrow \infty} l_{n}\left(l_{0}, w, u^{(\alpha)}\right) .
$$

It is easily seen, by scaling, that

$$
l_{n}\left(\delta l_{0}, \delta w\right)=\delta l_{n}\left(l_{0}, w\right)
$$


Let $\Omega_{n, \infty}$ be a infinite domain obtained by the periodic extension of $\Omega$ in the direction of $\mathbf{n}\left(\varphi^{-} \pm \pi / 2\right)$, and let $U_{n}\left(u_{n} ; \Omega_{n, \infty}\right)$ be the set of periodic extensions to $\Omega_{n, \infty}$ of all functions in $U_{n}\left(u_{n} ; \Omega\right)$. It is not difficult to see that for functions in $U_{n}\left(u_{n} ; \Omega_{n, \infty}\right)$ there are, in average, $w^{-1}$ twins on the left hand side boundary of $\Omega_{n, \infty}$ with respect to per unit width in $\mathbf{n}^{-}$direction.

Now, we consider a laminated needle-like microstructure as follows: Let $\Omega_{\infty}$ be a infinite domain similar to $\Omega_{n, \infty}$. On the right hand side of $\Omega_{\infty}$ it is a twin needles structure introduced by $U_{n}\left(u_{n} ; \Omega_{n, \infty}\right)$ with twin width $w$ and the length of the domain $\Omega_{n, \infty}$ in the direction perpendicular to $\mathbf{n}^{-}$being $l_{0}$, attached to the left is a simple laminated twin structure with twin width $w$ and length $l-\frac{1}{2} l_{0}$. Typically, the total length of a twin microstructure, measured from the left hand side boundary of $\Omega_{\infty}$ to the needle tip, is $l$. Let $\hat{l}_{0}=l_{0} w^{-1}$, and we call $\hat{l}_{0}$ the characteristic length of the needles. Let $E\left(l, \hat{l_{0}} ; w\right)$ be the average total potential energy of such a microstructure on per unit width of $\Omega_{\infty}$ in $\mathbf{n}^{-}$direction. We have, by summing up the surface energy and the elastic energy of the needles, and by using (3.8) and (3.10), that

$$
E\left(l, \hat{l}_{0} ; w\right)=2 l \sigma_{s} w^{-1}+\left(l_{n}\left(\hat{l}_{0}, 1\right)-1 \hat{l}_{0}\right) \sigma_{s}+E_{n}\left(\hat{l}_{0}, 1\right) w
$$

where $\sigma_{s}$ is the surface energy density which is assumed to be an elastic constant defined on twin boundaries as well as on needle boundaries.

For a given specimen with total twin microstructure length $l$, the energetic preferred twin laminated needle-like microstructure and the twin width is given by the solution of the following problem

$$
\left\{\begin{array}{l}
\text { find } w>0 \text { and } \hat{l}_{0} \in\left[\tau, 2 l w^{-1}\right] \text { such that } \\
E\left(l, \hat{l}_{0} ; w\right)=\inf _{\substack{v>0 \\
\tilde{l}_{0} \in\left[\tau, 2 l v^{-1}\right]}} E\left(l, \tilde{l}_{0} ; v\right),
\end{array}\right.
$$

where $\tau>0$ is a constant depend loosely on the elastic properties of the crystal. We do not search $\hat{l}_{0}$ in $\left(0, l w^{-1}\right]$, since in applications characteristic length of needles is generally much greater than 1 , and numerical computations show that the needle structure has great elastic energy and is unstable if $\hat{l}_{0}$ is too small.

Lemma 3.1. If $\left(\hat{l}_{0}, w\right)$ is a solution of problem (3.12), then

$$
w=\left(\frac{2 l \sigma_{s}}{E_{n}\left(\hat{l}_{0}, 1\right)}\right)^{\frac{1}{2}},
$$


and thus the total potential energy has the form

$$
\begin{aligned}
E\left(l, \hat{l}_{0}\right) & \equiv E\left(l, \hat{l}_{0} ;\left(\frac{2 l \sigma_{s}}{E_{n}\left(\hat{l}_{0}, 1\right)}\right)^{\frac{1}{2}}\right) \\
& =2\left(2 l \sigma_{s} E_{n}\left(\hat{l}_{0}, 1\right)\right)^{\frac{1}{2}}+\left(l_{n}\left(\hat{l}_{0}, 1\right)-\hat{l}_{0}\right) \sigma_{s}
\end{aligned}
$$

Proof. For a given $\hat{l}_{0}$, we have

$$
\frac{\partial E}{\partial w}\left(l, \hat{l}_{0} ; w\right)=-\frac{2 l \sigma_{s}}{w^{2}}+E_{n}\left(\hat{l}_{0}, 1\right) .
$$

Since $w$ minimizes $E\left(l, \hat{l}_{0} ; \cdot\right)$, we have $\frac{\partial}{\partial w} E\left(l, \hat{l}_{0} ; w\right)=0$ which gives the equation (3.13).

By attaching a simple twin laminate and a pure austenite to a twin needle structure on the left and right hand side boundaries respectively, and by inserting arbitrarily thin needles, of which the gradient is the average gradient of the twins, between the twin boundaries, we see that $E_{n}\left(\hat{l}_{0}, 1\right)$ is nonincreasing respect to $\hat{l}_{0}$.

Lemma 3.2. Let $E\left(l, \hat{l}_{0}\right)$ be defined by the formula (3.14). Then, the problem

$$
E\left(l, \hat{l}_{0}\right)=\inf _{\tilde{l} \in(\tau, \infty)} E(l, \tilde{l})
$$

has a solution, if

$$
0<\sigma_{s}<8 l\left(E_{n}^{\frac{1}{2}}(\tau+1,1)-E_{n}^{\frac{1}{2}}(\tau, 1)\right)^{2} .
$$

Proof. By the definition of $l_{n}\left(\hat{l}_{0}, 1\right)$, it is easily seen that

$$
2 \hat{l}_{0}<l_{n}\left(\hat{l}_{0}, 1\right)<2 \hat{l}_{0}+1
$$

and thus we have

$$
\lim _{\hat{l}_{0} \rightarrow+\infty} E\left(l, \hat{l}_{0}\right)=+\infty, \quad \text { if } \sigma_{s}>0
$$

On the other hand, if (3.17) is satisfied, it follows from (3.14) and (3.18) that

$$
E(l, \tau+1)-E(l, \tau)<2\left(2 l \sigma_{s}\right)^{\frac{1}{2}}\left(E_{n}^{\frac{1}{2}}(\tau+1,1)-E_{n}^{\frac{1}{2}}(\tau, 1)\right)+\sigma_{s}<0 .
$$

The relations (3.19), (3.20) and the continuity of $E(l, \cdot)$ implies the lemma.

Theorem 3.1. The problem (3.12) has at least one solution, if there exists a $\tau$ such that (3.17) is satisfied. 
Proof. By lemma 3.2, the problem (3.16) has at least one solution. Let $\hat{l}_{0} \in$ $(\tau, \infty)$ be a solution of the problem (3.16) and let $w$ be given by (3.13), suppose that the inequality

$$
l \geq \frac{2 \hat{l}_{0}^{2} \sigma_{s}}{E_{n}\left(\hat{l}_{0}, 1\right)}
$$

is satisfied, then we have, by direct calculation, that $\hat{l}_{0} \in\left[\tau, l w^{-1}\right]$ and thus $\left(\hat{l}_{0}, w\right)$ is a solution of the problem (3.12). On the other hand, if (3.21) is not satisfied by an absolute minimizer of the problem (3.16), then by comparing the energies of the local minimizers of (3.16), if there is any, and the energy corresponding to $\hat{l}_{0}$ satisfying the equality in $(3.21)$, we can still obtain a solution of the problem (3.12).

Theorem 3.2. Let $l$ and $\sigma_{s}$ be given. Suppose there is a $\tau$ such that (3.17) is satisfied. Let $\hat{l}_{0}$ be a solution of the problem (3.16) satisfying (3.21). Then, the twin width of the laminated microstructure is given by

$$
w=\left(\frac{2 l \sigma_{s}}{E_{n}\left(\hat{l}_{0}, 1\right)}\right)^{\frac{1}{2}},
$$

Proof. The conclusion of the theorem is a direct consequence of Theorem thm:3.1 and Lemma lem:3.1.

Remark 3.1. For needle-like twin laminates without branch, by the relation (3.22) (see also $(3.13))$ and that $E_{n}\left(\hat{l}_{0}, 1\right)$ is nonincreasing and has a positive lower bound, we see that for $l$ sufficiently large the twin width $w$ is asymptotically a linear function of $\left(l \sigma_{s}\right)^{1 / 2}$. However, for $l$ relatively small, the relation is not that simple.

\section{The MESh TRANSFORMATION METHOD}

Let $\Omega \subset R^{n}$ be a bounded open set with a Lipschitz continuous boundary $\partial \Omega$, $\partial \Omega_{0}$ is a subset of $\partial \Omega$ with positive measure. Let $f: R^{m n} \rightarrow R^{1}$ be a continuous function which satisfies the following hypotheses for a constant $p>1$ :

(h1): $\max \left\{a_{0}, a_{1}+b_{1}|\xi|^{p}\right\} \leq f(\xi) \leq a_{2}+b_{2}|\xi|^{p}$,

$$
\text { (h2): }|f(\xi)-f(\eta)| \leq C\left(1+|\xi|^{p-1}+|\eta|^{p-1}\right)|\xi-\eta| \text {, }
$$


where $a_{0}, a_{1} \in R^{1}, a_{2}>0, b_{2} \geq b_{1}>0$ and $C>0$ are constants. Consider the problem of minimizing the functional

$$
F(u ; \Omega)=\int_{\Omega} f(\nabla u(x)) d x
$$

on a set of admissible functions

$$
\mathbb{U}\left(u_{0} ; \Omega\right)=\left\{u \in W^{1, p}\left(\Omega ; R^{m}\right): u(x)=u_{0}(x) \text {, on } \partial \Omega_{0}\right\} .
$$

Define

$$
\begin{array}{r}
T(\Omega)=\left\{\text { bijections } L: \bar{\Omega} \rightarrow \bar{\Omega} \mid L \in W^{1, \infty}\left(\Omega ; R^{m}\right), L^{-1} \in W^{1, \infty}(\Omega),\right. \\
\left.L\left(\partial \Omega_{0}\right)=\partial \Omega_{0}, \text { and } \operatorname{det} \nabla L>0, \text { a.e. in } \Omega\right\} .
\end{array}
$$

For any $L \in T(\Omega)$ and $u \in \mathbb{U}\left(u_{0} ; \Omega\right)$, let $A \in R^{m n}$ and let $\bar{u}(x): \Omega \rightarrow R^{m}$ be defined by

$$
\bar{u}(x)=u(L(x))-A L(x) .
$$

Then it is easily seen that $\bar{u} \in \mathbb{U}\left(\bar{u}_{0} ; \Omega\right)$, where $\bar{u}_{0}(x)=u_{0}(L(x))-A L(x)$,

$$
\int_{\Omega} f\left(A+\nabla \bar{u}(x)(\nabla L(x))^{-1}\right) \operatorname{det} \nabla L(x) d x=F(u ; \Omega),
$$

and

$$
\inf _{\bar{u} \in \mathbb{U}\left(\bar{u}_{0} ; \Omega\right)} F(\bar{u}, L ; \Omega)=\inf _{u \in \mathbb{U}\left(u_{0} ; \Omega\right)} F(u ; \Omega)
$$

where

$$
\left.F(\bar{u}, L ; \Omega)=\int_{\Omega} f\left(A+\nabla \bar{u}(x)(\nabla L(x))^{-1}\right)\right) \operatorname{det} \nabla L(x) d x .
$$

Let $\mathfrak{T}_{h}(\Omega)$ be regular triangulations of $\Omega$ with mesh size $h$ [23]. Let

$$
\begin{aligned}
& T_{h}(\Omega)=\left\{L \in T(\Omega):\left.L\right|_{K} \text { is affine } \forall K \in \mathfrak{T}_{h}(\Omega)\right\}, \\
& \mathbb{U}_{h}=\left\{u \in(C(\bar{\Omega}))^{m}:\left.u\right|_{K} \text { is affine } \forall K \in \mathfrak{T}_{h}(\Omega)\right\}
\end{aligned}
$$

and

$$
\mathbb{U}_{h}(v ; \Omega)=\left\{u \in \mathbb{U}_{h}:\left.u\right|_{\partial \Omega_{0}}=v\right\} .
$$

Instead of finding a minimizer of $F(u ; \Omega)$ in a fixed finite element function space $\mathbb{U}_{h}\left(u_{0} ; \Omega\right)$, we solve the following discrete problem :

$$
(M T M)\left\{\begin{array}{l}
\text { find }\left(\bar{u}_{h}, L_{h}\right) \in \mathbb{U}_{h}\left(\bar{u}_{0} ; \Omega\right) \times T_{h}(\Omega) \text { such that } \\
F\left(\bar{u}_{h}, L_{h} ; \Omega\right)=\inf _{\left(u^{\prime}, L^{\prime}\right) \in \mathbb{U}_{h}\left(\bar{u}_{0} ; \Omega\right) \times T_{h}(\Omega)} F\left(u^{\prime}, L^{\prime} ; \Omega\right),
\end{array}\right.
$$


and use its solution $\left(\bar{u}_{h}, L_{h}\right)$ and the relation (4.3) to construct a finite element solution

$$
u_{h}(x)=\bar{u}_{h}\left(L_{h}^{-1}(x)\right)+A x .
$$

This is the so called mesh transformation method (for a more general form and the convergence analysis of the mesh transformation method, see $[8,9])$, which by (4.3)-(4.5) is equivalent to minimizing the elastic energy $F(\cdot ; \Omega)$ among all finite element function spaces introduced by $\mathfrak{T}_{h}(\Omega)$ and $T_{h}(\Omega)$, and thus is capable of producing much better numerical results than working on a fixed finite element function space.

In our application, the domain $\Omega$ is taken to be a parallelogram with sides of length $2 a$ and $2 b$ perpendicular to $\mathbf{n}(\alpha \pm \pi / 2)$ and $\mathbf{n}(\beta)$ respectively, where $\mathbf{n}(\tau)=$ $(\cos (\tau), \sin (\tau))^{T}$. More precisely, $\Omega=L_{0}(\alpha, \beta) D$ where $D=(-a, a) \times(-b, b)$ is a $2 a \times 2 b$ rectangular domain, $L_{0}(\alpha, \beta)$ is defined by

$$
L_{0}(\alpha, \beta)\left(\begin{array}{l}
x \\
y
\end{array}\right)=R(\alpha)\left(\begin{array}{c}
x+y \tan (\alpha-\beta) \\
y
\end{array}\right),
$$

and

$$
R(\alpha)=\left(\begin{array}{rr}
\cos \alpha & -\sin \alpha \\
\sin \alpha & \cos \alpha
\end{array}\right)
$$

is a rotational matrix.

Assume that the angles $\alpha$ and $\beta$ are so chosen that the laminated microstructure is periodically defined on $\Omega$ along the direction $\mathbf{n}(\beta \pm \pi / 2)[10,21]$, and thus the problem is equivalent to compute, on a domain which is infinite in the directions $\pm L_{0}(\alpha, \beta)(0, b)^{T}$, a solution which is periodic in the $\mathbf{n}(\beta \pm \pi / 2)$ with period $2 b / \cos (\alpha-\beta)$. In such a case, the mesh transformation map $L$ and the deformation $\bar{u}$ are naturally asked to satisfy the periodic condition:

$$
\begin{aligned}
(L-I)\left(L_{0}(\alpha, \beta)\left((x, b)^{T}\right)\right) & =(L-I)\left(L_{0}(\alpha, \beta)\left((x,-b)^{T}\right)\right), \forall x \in[-a, a], \\
\bar{u}\left(L_{0}(\alpha, \beta)(x, b)^{T}\right) & =\bar{u}\left(L_{0}(\alpha, \beta)(x,-b)^{T}\right), \quad \forall x \in[-a, a],
\end{aligned}
$$

where $I$ is the identity map.

The above periodic relaxation of the mesh transformation method allows us to focus our computation on a couple of twins and thus dramatically reduce the computing cost (the analysis and other applications of the periodic relaxation 
method can be found in $[9,10,11])$. The convergence analysis of the mesh transformation method can be found in $[8,9,11]$.

\section{NumERICAL EXPERIMENTS AND RESUltS}

Let $f(\nabla u ; \theta)$ be given by $(2.3)$ and (2.4). For a given total twin microstructure length $l$ and a surface energy density $\sigma_{s}$, to calculate the twin width of the twin laminate, by Theorem 3.2 and (3.14), we need to know the functions $E_{n}\left(\hat{l}_{0}, 1\right)$ and $l_{n}\left(\hat{l}_{0}, 1\right)$. To establish the functions numerically, we first solve the problem (3.6) by the mesh transformation method given in section 4 for a set of discrete data $\left(l_{0}, w\right)$, and then fit the numerical results with some properly chosen curves by using the least square method.

Let $\alpha=\frac{\pi}{4}$ and $\beta=\varphi^{-}$which is given by (3.2). Let $\Omega=L_{0}(\alpha, \beta) \hat{\Omega}$, where $\hat{\Omega}=(-a, a) \times(-b, b)$ is a $2 a \times 2 b$ rectangular domain and $L_{0}(\alpha, \beta)$ is defined by (4.12). Let $\mathfrak{T}_{N, M}(\Omega)=\mathfrak{T}_{h}(\Omega)$ be a family of regular triangulations defined by

$$
\mathfrak{T}_{N, M}(\Omega)=\mathfrak{T}_{h}(\Omega)=\mathfrak{T}_{h}\left(L_{0}(\alpha, \beta) \hat{\Omega}\right)=\left\{L_{0}(\alpha, \beta) K: \forall K \in \mathfrak{T}_{h}(\hat{\Omega})\right\},
$$

where $\mathfrak{T}_{h}(\hat{\Omega})=\mathfrak{T}_{N, M}(\hat{\Omega})$, for $h=h_{N, M}=\frac{2}{N \cdot M} \sqrt{(a M)^{2}+(b N)^{2}}$ with $N \geq 2$ and $M \geq 2$, is a family of regular triangulations of $\hat{\Omega}$ introduced by the lines

$$
\left\{\begin{array}{llrl}
y & =-b+\frac{2 b}{M} i, & & 0 \leq i \leq M \\
x & =-a+\frac{2 a}{N} j, & & 0 \leq j \leq N ; \\
y & =\frac{N b}{M a}(x+a)+b-\frac{4 b}{M} k, & & 0<k<\frac{M+N}{2} \\
y & =\frac{-N b}{M a}(x-a)-b+\frac{4 b}{M} k, & & 0<k<\frac{M+N}{2} .
\end{array}\right.
$$

Let

$$
\partial \Omega_{ \pm}=\left\{x \in \partial \Omega: x=L_{0}(\alpha, \beta)( \pm a, \eta)^{T},-b \leq \eta \leq b\right\}
$$

and

$$
\partial \Omega^{ \pm}=\left\{x \in \partial \Omega: x=L_{0}(\alpha, \beta)(\xi, \pm b)^{T},-a \leq \xi \leq a\right\} .
$$

Let the temperature distribution $\theta(x)$ be a linear function given by

$$
\theta(x)=\theta\left(L_{0}(\alpha, \beta)(\xi, \eta)^{T}\right)=\theta_{-}+\frac{\theta_{+}-\theta_{-}}{2 a}(\xi+a),
$$

which takes the value of $\theta_{ \pm}$on $\partial \Omega_{ \pm}$.

In the following numerical experiments, we set the width of the reference configuration $2 b=0.1$, and set the elastic constants $\alpha=2.02 / \pi, \mu=0.25, \gamma=0$, $\hat{\varepsilon}=0.05, d_{0}=500, e=3.5$ and $g=15$ (see (2.4)-(2.7)). The initial temperature 
distribution $\theta(x)$ is given by (5.5) with $\theta_{-}=69.65^{\circ} \mathrm{C}$ and $\theta_{+}=70.95{ }^{\circ} \mathrm{C}$, while the transformation temperature is taken to be $\theta_{T}=70^{\circ} \mathrm{C}$. The initial mesh is introduced by taking $M=4$ and $N=2$. The initial deformation $u_{0}(x)$ is introduced in the following way: let the boundary value $u_{n}(x)$ be given by $(3.5)$ with $w=2 b=0.1$, let $u_{0}(x) \in \mathfrak{T}_{2,4}(\Omega)$, which is a piecewise linear finite element function defined on a mesh with $M=4$ and $N=2$, satisfy $u_{0}(x)=u_{n}(x)$ for all $x \in \partial \Omega_{ \pm}$and $u_{0}(x)=R\left(\xi^{-}\right)\left(x-w\left(\frac{1}{2}-\lambda\right) \mathbf{n}^{-}\right)$if $\theta(x) \geq \theta_{T}$. The volume fraction $\lambda$ is taken to be 0.5 in our numerical experiments.

For a fixed needle length $l_{0} / 2=a$, the mesh transformation method described in section 4 is applied to solve the discrete problem of (3.6), and the conjugate gradient method is used to search for a minimizer. To avoid the elements being deformed too much and too fast where the initial deformation gradient is well away from the wells, the mesh transformation map $L$ is kept fixed in the beginning of the minimizing process until the drop of the elastic energy is getting lost of its initial momentum. To guarantee that the condition $\operatorname{det} \nabla L(x)>0$ is satisfied in the minimization process, it is checked on each element in the linear search along the direction given by the conjugate gradient method and the step length is reduced whenever necessary. When certain convergence criteria (say $\left.\|\nabla F\|_{2}<0.25 \times 10^{-6}\right)$ is satisfied, the search is continued with the mesh being refined by a factor of 4 until the mesh is sufficiently fine. In our numerical experiments, the finest mesh is set to be $M=64$ and $N=32$. Then, the temperature distribution $\theta(x)$ is set to be a uniform distribution at the transformation temperature $\theta_{T}$, and the minimizer is searched by the conjugate gradient method with the finite element functions $\left(\bar{u}_{h, \theta}, L_{h, \theta}\right)$ produced by the above computation as the initial data. The numerical results thus obtained is denoted by $\left(\bar{u}_{h, \theta_{T}}, L_{h, \theta_{T}}\right)$. By section 4 , the final mesh defined on the material reference configuration $\Omega$ is

$$
\mathfrak{T}_{h, \theta_{T}}(\Omega) \equiv \mathfrak{T}_{h}\left(L_{h, \theta_{T}} \Omega\right)=\left\{L_{h, \theta_{T}} K: \forall K \in \mathfrak{T}_{h}(\Omega)\right\},
$$

by (4.11) and by taking $A=A_{\lambda}^{-}$, the finite element minimizing deformation is given by

$$
u_{h, \theta_{T}}(x)=\bar{u}_{h, \theta_{T}}\left(L_{h, \theta_{T}}^{-1}(x)\right)+A_{\lambda}^{-} x .
$$

A typical convergence behavior of the minimization procedure with characteristic needle length $\hat{l}_{0}=7.5$ is shown in figure 3 , where in figure-3(a) (figure-3(b)) the convergence behavior before and after the first (second) mesh refinement point 
is shown. We see that the energy reduction before the first mesh refinement point is about $1.9 \times 10^{-5}$, and that between the first and second mesh refinement points and that after the second mesh refinement point are $6.2 \times 10^{-6}$ and $5.4 \times 10^{-7}$ respectively. This clearly shows the convergence of the procedure.
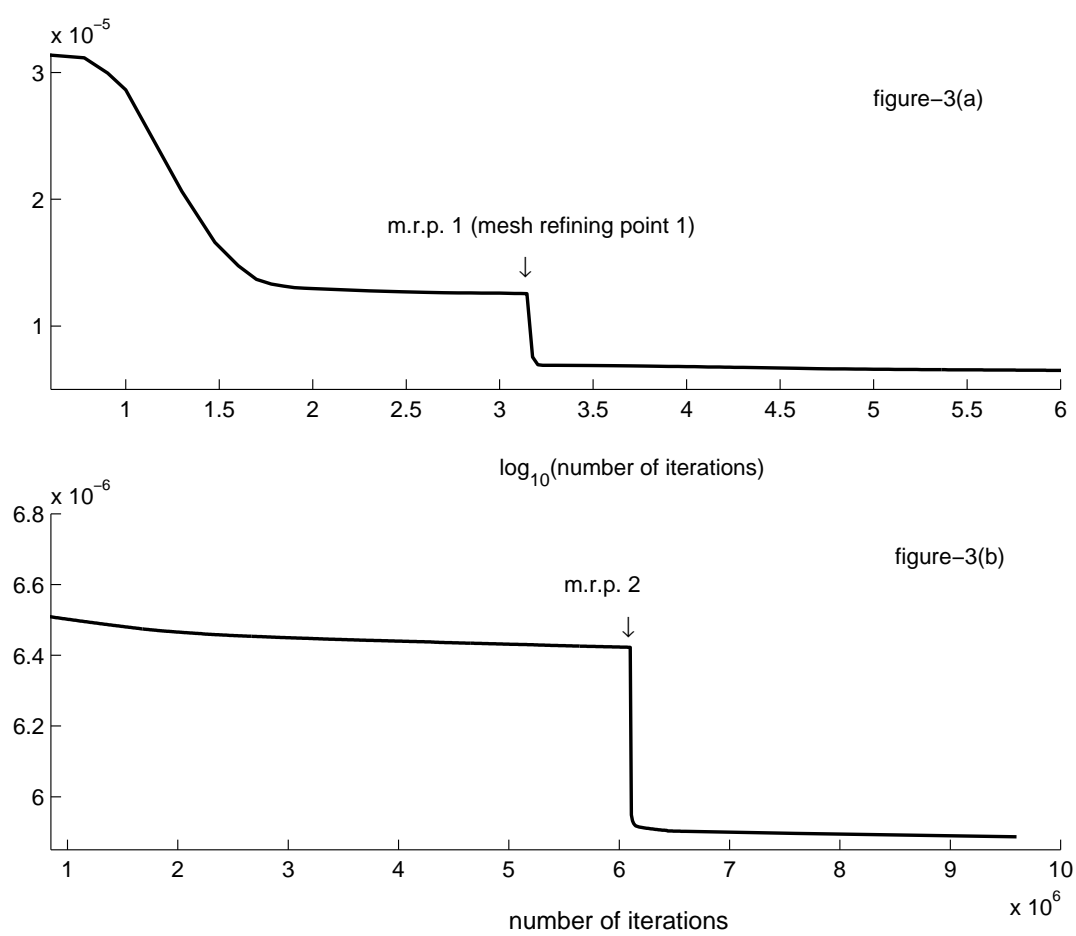

FIGURE 3. The convergence of the minimization procedure.

Some typical profiles of the needles on the deformed configuration for various characteristic needle lengths $\hat{l}_{0}=a b^{-1}$ are shown in figure 4 , where $x_{1}$ and $y_{1}$ are the length scales corresponding to the directions $\mathbf{n}(\pi / 4)$ and $\mathbf{n}(-\pi / 4)=$ $\mathbf{n}^{-}$respectively, and where an element is painted in white, black or grey if its deformation gradient is closer to $U_{0}, R^{-} U_{1}$ or $R\left(\xi^{-}\right)$respectively. It is clearly seen that, as the characteristic length of the needles increases, the needles change their shape from strictly concave to partially convex and finally grow into the twin laminate near the flat end of the needles.

The numerical results of $E_{n}\left(a b^{-1}, 1\right)$ and $l_{n}\left(a b^{-1}, 1\right)$ at some discrete points are shown in table 1.

Next, we establish the approximate functions of $E_{n}\left(\hat{l}_{0}, 1\right)$ and $l_{n}\left(\hat{l}_{0}, 1\right)$. Since the numerical results show that the value of $E_{n}\left(\hat{l}_{0}, 1\right)$ drops quite fast to its limit 

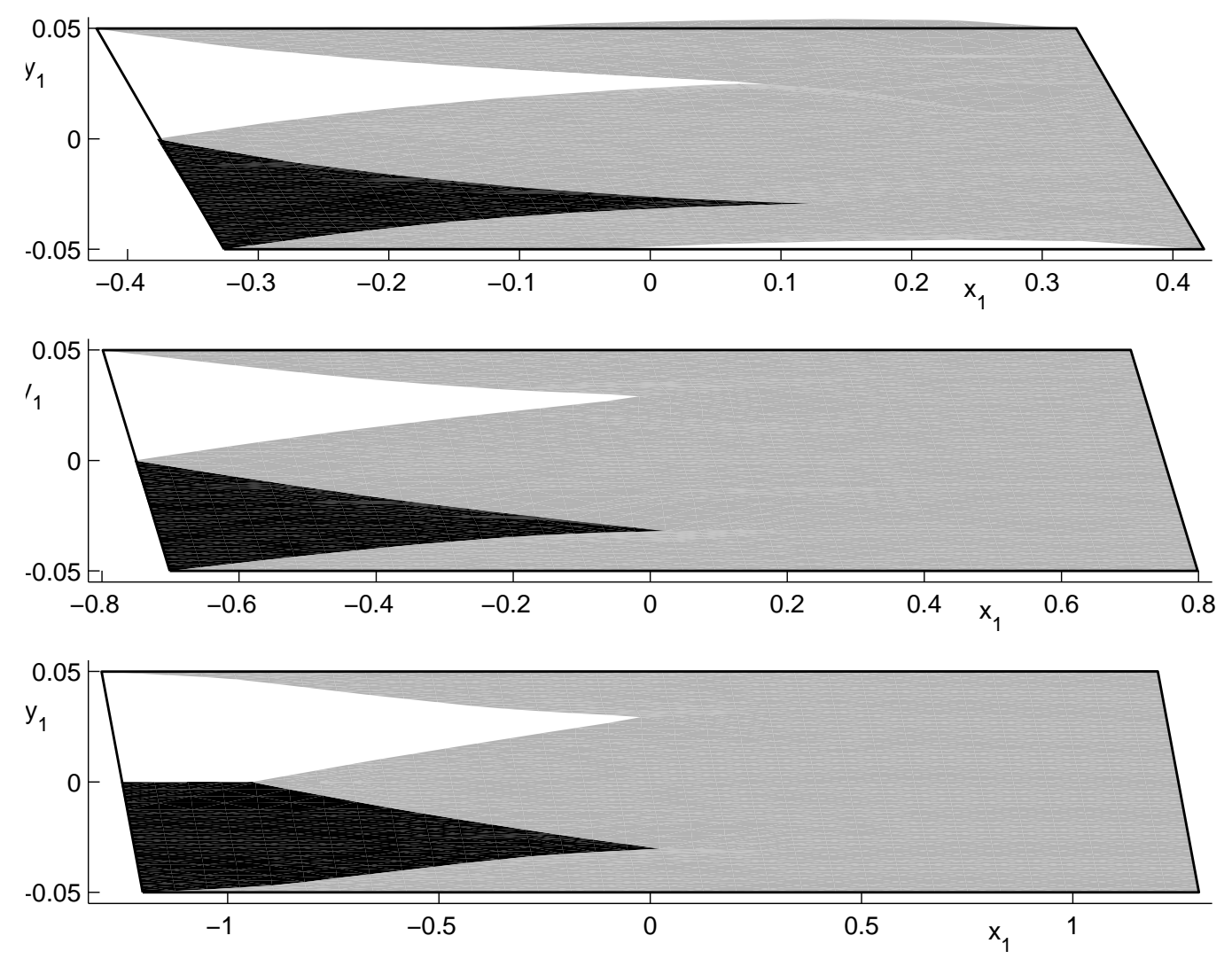

FiguRE 4. The needle profiles for $\hat{l}_{0}=7.5,15,25$ respectively.

TABLE 1. Some numerical results of $E_{n}\left(a b^{-1}, 1\right)$ and $l_{n}\left(a b^{-1}, 1\right)$.

\begin{tabular}{|c|c|c|c|c|c|c|}
\hline$a b^{-1}$ & 7.5 & 10.0 & 12.5 & 15.0 & 17.5 & 20.0 \\
\hline$E_{n}$ & $6.22 \times 10^{-4}$ & $4.14 \times 10^{-4}$ & $3.14 \times 10^{-4}$ & $2.72 \times 10^{-4}$ & $2.43 \times 10^{-4}$ & $2.33 \times 10^{-4}$ \\
\hline$l_{n}$ & 17.015 & 21.375 & 21.397 & 23.601 & 27.875 & 31.077 \\
\hline
\end{tabular}

as $\hat{l}_{0}$ increases, we consider it to be an exponential function of the form

$$
E_{n}\left(\hat{l}_{0}, 1\right)=E_{n}(\infty, 1)+\exp \left(c_{1}-c_{2} \hat{l}_{0}\right)
$$

where $E_{n}(\infty, 1)=\lim _{\hat{l}_{0} \rightarrow \infty} E_{n}\left(\hat{l}_{0}, 1\right), c_{1} \in R^{1}$ and $c_{2}>0$ are material constants to be determined. Figure 5(a) shows the numerical approximation of the function $E_{n}\left(\hat{l}_{0}, 1\right)$, with $E_{n}(\infty, 1)=2.243 \times 10^{-4}, c_{1}=-5.522$ and $c_{2}=0.3075$, obtained by applying the nonlinear least square method to some discrete numerical results of $E_{n}\left(\hat{l}_{0}, 1\right)$, which are shown as $* \mathrm{~s}$ in the figure, with $l^{2}$-norm of the error $1.1 \times 10^{-5}$. For $l_{n}\left(\hat{l}_{0}, 1\right)$, we simply use a cubic spline to fit the discrete numerical 
results. Figure $5(\mathrm{~b})$ shows the numerical approximation of $l_{n}\left(\hat{l}_{0}, 1\right)$ by using the cubic spline interpolation with not-a-knot boundary condition. With the functions $E_{n}\left(\hat{l}_{0}, 1\right)$ and $l_{n}\left(\hat{l}_{0}, 1\right)$ known, the function $E\left(l, \hat{l}_{0}\right)$ defined by $(3.14)$ is then, for a given total twin microstructure length $l$ and a given surface energy density $\sigma_{s}$, a function of $\hat{l}_{0}$. Figure $5(\mathrm{c})$ shows the function $E\left(l, \hat{l}_{0}\right)$ with $l=1$ and $\sigma_{s}=10^{-8}$, which obviously has an unique minimizer satisfying the inequality $(3.21)$.
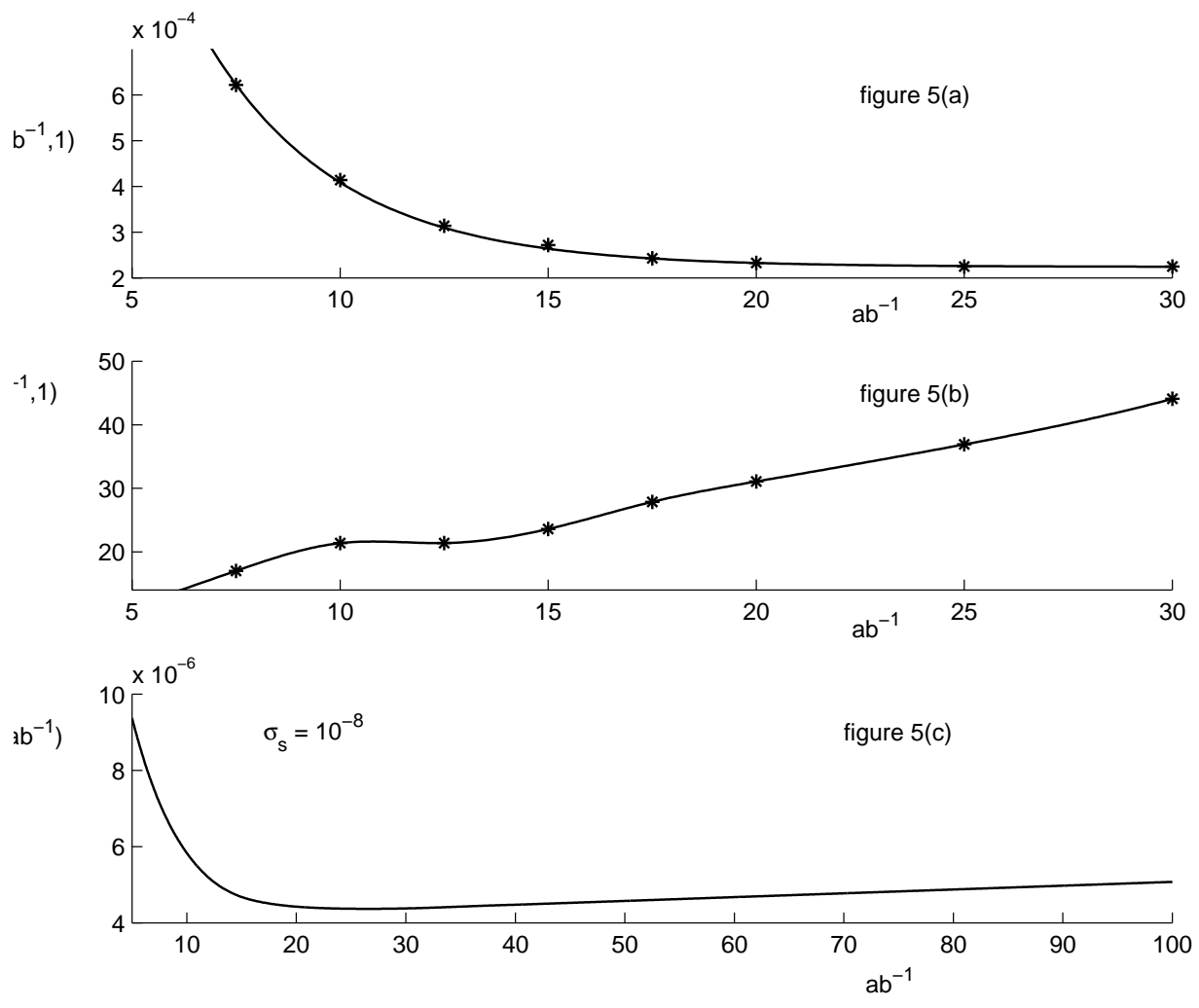

FiguRE 5 . The approximate curves of $E_{n}\left(\hat{l}_{0}, 1\right), l_{n}\left(\hat{l}_{0}, 1\right)$ and the resulting approximate function $E\left(l, \hat{l}_{0}\right)$ with $l=1.0$ and $\sigma_{s}=10^{-8}$.

In fact, the numerical experiments show that the inequality (3.21) is always satisfied by the unique minimizer of $E\left(l, \hat{l}_{0}\right)$ for various total length $l$ of the twin laminates and the surface energy density $\sigma_{s}$ satisfying the inequality (3.17) where $\tau$ can be set to 7.0. Thus, by theorem 3.2, the twin width of the laminates is given by

$$
w=\left(\frac{2 l \sigma_{s}}{E_{n}\left(\hat{l}_{0}, 1\right)}\right)^{\frac{1}{2}}
$$


In figure 6 , the relation between the twin width $w$ and the total length of the twin laminate $l$ for various surface energy density $\sigma_{s}$ is shown, where $\left\{\sigma_{s}^{i}\right\}_{i=1}^{8}=$ $\left\{2 \times 10^{-10}, 8 \times 10^{-10}, 2 \times 10^{-9}, 4 \times 10^{-9}, 7 \times 10^{-9}, 1.1 \times 10^{-8}, 1.8 \times 10^{-8}, 3 \times 10^{-8}\right\}$ respectively. It is clearly seen that the twin width $w$ is approximately a linear function of $l^{1 / 2}$, of which the slope is an increasing function of the surface energy density $\sigma_{s}$.

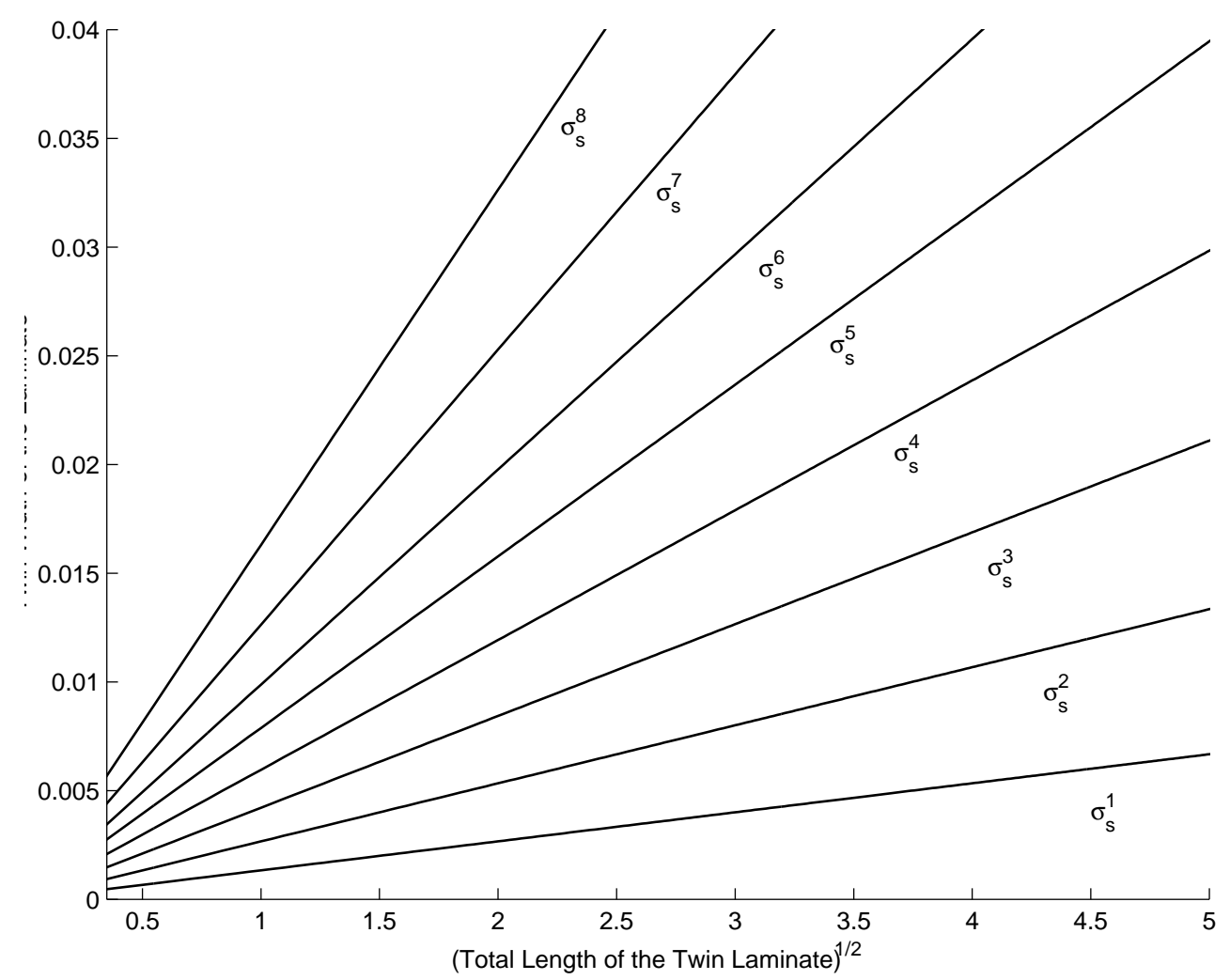

FiguRE 6 . The relation between the twin width $w$ and the total twin length $l$ for various surface energy density $\sigma_{s}$.

In figure 7 , the relation between the characteristic needle length $\hat{l}_{0}$ and the total length of the twin laminate $l$ for various surface energy density $\sigma_{s}$ is shown, where the $i$ 'th curve's surface energy density is $\sigma_{s}^{i}$ with $\left\{\sigma_{s}^{i}\right\}_{i=1}^{8}=\left\{2 \times 10^{-10}, 8 \times\right.$ $\left.10^{-10}, 2 \times 10^{-9}, 4 \times 10^{-9}, 7 \times 10^{-9}, 1.1 \times 10^{-8}, 1.8 \times 10^{-8}, 3 \times 10^{-8}\right\}$ respectively. Since the characteristic needle length $\hat{l}_{0}=l_{0} w^{-1}$ shows the sharpness of the needles, we see in figure 7 that the increase of $l$ sharpens the needle only slightly especially when $l>10$. 


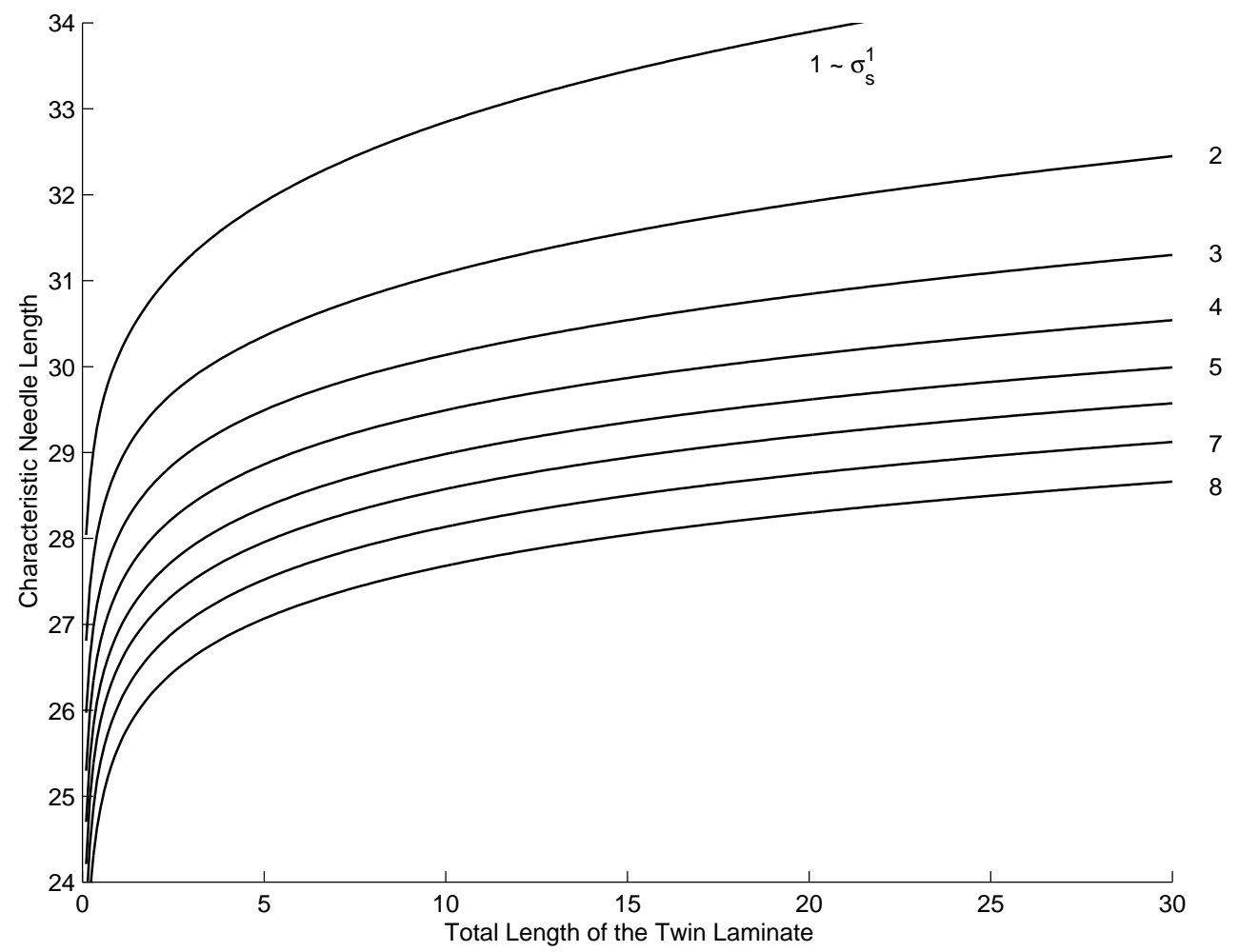

Figure 7 . The relation between the characteristic needle length $\hat{l}_{0}$ and the total twin length $l$ for various surface energy density $\sigma_{s}$.

By figure 7 , we see that the characteristic needle length $\hat{l}_{0}$ varies only slightly in a neighborhood of 28 for different $l$ and $\sigma_{s}$. Thus, by figure 5(a), the needle's elastic energy density $E_{n}\left(\hat{l}_{0}, 1\right)$ is almost a constant for different $l$ and $\sigma_{s}$. This and the relation (5.9) imply that the twin width $w$ is also approximately a linear function of $\sigma_{s}^{1 / 2}$, of which the slope is approximately a linear function of $l^{1 / 2}$.

As a conclusion of our theory and numerical experiments, we have that, for a needle-like laminated microstructure without branch, the twin width $w$ is approximately related to the twin length $l$ and the surface energy density $\sigma_{s}$ by

$$
w=C\left(l \sigma_{s}\right)^{\frac{1}{2}},
$$

where $C$ is a material constant which in our case is about 94.28. Hence the well known prediction (5.10) [1] is a simplified version of our theory, and the material constant can be obtained numerically by the method given in this paper. 


\section{REFERENCES}

[1] J. M. Ball and R. D. James, Fine phase mixtures as minimizers of energy. Arch. Rat. Mech. Anal., 100(1987)1, 13-52.

[2] J. M. Ball and R. D. James, Proposed experimental test of a theory of fine microstructure and the two-well problem. Phil. Trans. R. Soc. London 338A(1992), 389-450.

[3] M. Chipot and D. Kinderlehrer, Equilibrium configurations of crystals, Arch. Rat. Mech. Anal., 103(1988)1, 237-277.

[4] D. Kinderlehrer, Remarks about equilibrium configurations of crystals, in Material Instabilities in Continuum Mechanics and Related Problems, J. M. Ball, ed., Oxford University press, 1987, pp. 217-242.

[5] R.V. Kohn and Stefan Müller, Branching of twins near an austenite-twinned-martensite interface. Phil. Mag., A 66(1992), 697-715.

[6] R.V. Kohn and Stefan Müller, Surface energy and microstructure in coherent phase transitions. Comm. Pure Appl. Math., 47(1994), 405-435.

[7] Z.-P. Li, Rotational transformation method and some numerical techniques for the computation of microstructures. Math. Models Meth. Appl. Sci., 8(1998), 985-1002.

[8] Z.-P. Li, A mesh transformation method for computing microstructures. To appear in Numer. Math..

[9] Z.-P. Li, A periodic relaxation method for computing microstructures. Appl. Numer. Math., 32(2000), 291-303.

[10] Z.-P. Li, Computations of needle-like microstructures, Toappear in Appl. Numer. Math..

[11] Z.-P. Li, Mesh re-regularization and quasi-static numerical simulation of austeniticmartensitic phase transition. Research Report No.11, Institute of Mathematics and School of Mathematical Sciences, Peking University, 2000.

[12] C. Collins, Computation of twinning, in Microstructure and Phase Transitions, IMA Volumes in Mathematics and Its Applications, Vol.54, J. Ericksen, R. James, D. Kinderlehrer and M. Luskin, eds, Springer-Verlag, New York, (1993), pp. 39-50.

[13] C. Collins, M. Luskin and J. Riordan, Computational results for a two-dimensional model of crystalline microstructure, in Microstructure and Phase Transitions, IMA Volumes in Mathematics and Its Applications, Vol.54, J. Ericksen, R. James, D. Kinderlehrer and M. Luskin, eds, Springer-Verlag, New York, (1993), pp. 51-56.

[14] M. Luskin, On the computation of crystalline microstructure. Acta Numerica, 5(1996), 191-257.

[15] Z.-P. Li, Laminated microstructure in a variational problem with a non-rank-one connected double well potential, J. Math. Anal. Appl., 217(1998), 490-500.

[16] J.M. Ball, A version of the fundamental theorem for Young measures. In Partial Differential Equations and Continuum models of Phase Transitions, Lecture Notes in Physics, No. 344, M. Rascle, D. Serre and M. Slemrod, eds., Springer-Verlag, New York (1989), pp. 207-215. 
[17] P. G. Ciarlet, Mathematical Elasticity, Volume 1: Three Dimensional Elasticity. NorthHolland, Amsterdam, 1988.

[18] P. Klouček and M. Luskin, The computation of the dynamics of the martensitic transformation, Continuum Mech. Thermodyn., 6(1994), 209-240.

[19] C. Collins and M Luskin, The computation of the austenitic-martensitic phase transition. In Partial Differential Equations and Continuum Models of Phase Transitions Lecture Nodes in Physics, Vol. 344, M. Rascle, D. Serre and M. Slemrod, eds, SpringerVerlag, New York, (1989), pp. 34-50.

[20] Z.-P. Li, Finite order rank-one convex envelopes and computation of microstructures with laminates in laminates. BIT Numer. Math., 40(4)(2000), pp.745-761.

[21] B. Li and M. Luskin, Theory and computation for the microstructure near the interface between twinned layers and a pure variant of martensite. Materials Sci. Eng. A, 1999.

[22] R. Abeyaratne, C. Chu and R. D. James, Kinetics of materials with wiggly energies: the evolution of twinning microstructure in a Cu-Al-Ni shape memory alloy. Phil. Mag. 73A (1996), 457-497.

[23] P.G. Ciarlet, The Finite Element Method for Elliptic Problems. North-Holland, Amsterdam, 1978.

E-mail address: zpli@math.pku.edu.cn 